\title{
CA-MWSN: CluSTERING AlgORITHM FOR MOBILE WIRELESS SENOR NETWORK
}

\author{
Priti Kumari $^{1}$ \\ ${ }^{1}$ Department of Computer Science Engineering, Netaji Subash Institute of Technology, \\ Bihta, Patna, Bihar, India
}

\begin{abstract}
This paper proposes a centralized algorithm for cluster-head-selection in a mobile wireless sensor network. Before execution of algorithm in each round, Base station runs centralized localization algorithm whereby sensors update their locations to base station and accordingly Base station performs dynamic clustering. Afterwards Base station runs CA-MWSN for cluster-head-selection. The proposed algorithm uses three fuzzy inputs Residual energy, Expected Residual Energy and Mobility to find Chance of nodes to be elected as Cluster-head. The node with highest Chance is declared as a Cluster-head for that particular cluster. Dynamic clustering provides uniform and significant distribution of energy in a non-uniform distribution of sensors. CA-MWSN guarantees completion of the round.
\end{abstract}

\section{KEYWORDS}

Mobile wireless sensor network, fuzzy logic, cluster-head, CA-MWSN, centralized algorithm, dynamic clustering

\section{INTRODUCTION}

Sensor networks have a deep impact on our day-to-day life. These are quite promising for the convenience of human and risk management. Another significant factor for their omnipresence in our life is 'Safety'; Safety of human beings or their belongings or animals or climate. Sensors have been mounted everywhere for the significant task of monitoring. And in such scenario Mobile wireless sensor network has fascinated and eased our life. In Consequence, Mobile wireless sensor network is getting distinct hot research areas these days like node localization and discovery algorithms [1] techniques for energy utilization and conservation, resource allocation and optimization, distributed deployment strategies, cognitive radio techniques ,dynamic adjusting strategies, network coding and its applications, network capacity, outage probability, performance analysis and optimization, MAC/network layer cooperative communications, security and privacy [2]. Among these research areas, energy conservation is one of the prominent challenges as sensor lacks the battery recharging or changing options, simultaneously they are supposed to operate for long network lifetime [3] like habitat monitoring requires months of monitoring. For supervising long term degradation of civil engineering structures, wireless sensors are supposed to communicate over several years [4]. In order to maximize the total battery life of a wireless network, we must minimize the energy consumption of the entire network. Clustering of sensor nodes, data-driven approaches [5], scheduling sleep intervals for extraneous nodes [6] are efficient ways of saving energy in sensor network. Earlier experiments show that Clustering of sensor nodes saves energy by 8 times [7]. Furthermore, cluster-based 
International Journal on Applications of Graph Theory in Wireless Ad hoc Networks and Sensor Networks(GRAPH-HOC) Vol.6, No.4, December 2014

sensor network is considered here among various wireless sensor network topology like clusterbased sensor network, sensor network with a fusion center, and concatenated sensor network.

This paper considers hypothesis and the design of a Clustering Algorithm in Mobile Wireless Sensor Network (CA-MWSN). Base station executes centralized localization algorithm [8] for performing dynamic clustering then CA-MWSN is run. After successful completion of each round of cluster-head localization algorithm is run and subsequently CA-MWSN. With the help of input variables and fuzzy inference engine, a node within a cluster is decided as the clusterhead. Before designing any clustering algorithm, numerous issues need to be pondered and these issues significantly differ from the underlying network. Like in homogenous network, the role of cluster-head certainly is transferred from one sensor node to another sensor node to avoid energy shortage of an individual node but in case of heterogeneous network one sensor node can perform the duty of cluster-head more rounds than others. In case of mobile wireless sensor networks, topology of network is supposed to change, as in our case all the sensor nodes including clusterheads are movable. In such scene, cluster-radius and location of cluster-head are thought to be decided dynamically looking over current positions of sensor nodes. And this gives uniform distribution of cluster-heads and better coverage either locally and globally. In case of Leach [7], if randomly generated probability did not lie below threshold, no cluster-head is being chosen. This vagueness is overcome in CA-MWSN by definite selection of cluster-head in each round until expected residual energy of all nodes in network go below to 'low'.

The rest of this paper is organized as follows. Section 2 gives the idea of working of algorithms previously designed for mobile wireless sensor network. Section 3 tells about pre-conditions required before executing CA-MWSN algorithm. Section 4 describes the premise of algorithm CA-MWSN. Section 5 concludes the paper and gives some prospects for future work.

\section{RELATED WORK}

Evolving needs of human being has speeded up researches in the area of mobile wireless sensor network. Though techniques and yields grew up but challenges also got multiplied as mobility of nodes came into existence. In the context of clustering algorithms for mobile wireless sensor network, some algorithms are advancement of low-energy adaptive clustering hierarchy LEACH [7], like LEACH-M (Leach-Mobile) [9] and LEACH-ME (Leach-Mobile- Enhanced) [10]. LEACH-M [9] is similar to LEACH [7] except the fact that sensor nodes are mobile and due to mobility sensors change their position and sometimes their clusters also. Hence before transmitting data, each sensor node confirms about its cluster-head firstly. And cluster heads removes mobile nodes from their node list if no data is received in continuous two time slots. In Leach-ME [Kumar et al. 2008], before election of cluster-head mobility factor of node is seen. Mobility factor is calculated in terms of remoteness where remoteness is distance between two nodes. Mobility factor, energy level and transition count are decision factors for selection of cluster-head. Apart from Leach-based protocols, there is LUDC (Location- Unaware distributed clustering algorithm for mobile wireless sensor networks using fuzzy logic) [11] in which clusterhead of a node is selected on the basis of four fuzzy descriptors: energy level, mobility, quality of link and received signal strength. There is another algorithm ACE-L (Algorithm for Cluster-head Election with Location) [12] which are suitable for pre-defined mobile wireless sensor network. For the selection of cluster-head, location of node is considered as it can be anticipated by name. Cluster-heads are chosen corresponding to some fixed reference points. But in case of gathering of sensor nodes at one place which is possible as nodes are movable, one cluster-head corresponding to one reference point may give lower performance. 
International Journal on Applications of Graph Theory in Wireless Ad hoc Networks and Sensor Networks(GRAPH-HOC) Vol.6, No.4, December 2014

Hitherto, algorithms are not discerning the real problem or property of mobile wireless sensor network i.e; movement of sensor nodes which should be considered firmly during design of an algorithm because logistics changes holistically in case of random motion probability. Scenario change in following respects:

1. If sensors become dense in particular area, more number of cluster-heads is needed than earlier.

2. If sensors become sparse in particular area, one cluster-head is able to cover larger area than earlier.

This paper tries to resolve issues created with non-uniform distribution of sensor nodes with proper energy usage. The idea is same as dynamic cell sectorization [13] in cellular communication. Most of the calculations are performed at the Base Station which is considered as source of infinite energy.

\section{PRE-CONDITIONS}

1. All sensors in the network are homogeneous and energy constrained.

2. Sensors and cluster-heads are movable.

3. Base station is static and placed far from the intended area.

4. Each sensor is equipped with location finding system [14] or network contains anchor nodes.

5. After selection of cluster-head, only cluster-heads communicate with base station.

Before execution of algorithm, it is assumed that all sensors must unicast their location information to Base Station and on judging the location of all nodes in network; Base Station divides the network into certain clusters dynamically. For each cluster, a cluster-head is decided. Dynamic clustering is inevitable due to following causes:

1. Ad-hoc deployment of sensor nodes- Sensors are deployed randomly, so clusters should be made after knowing the locations of sensor nodes.

2. Mobility of sensors- Sensors are free to move anywhere, as if there is accumulation of nodes cluster-radius is made sort.

3. Better coverage and energy balance because base station keeps global knowledge of network.

Once network gets divided into clusters, sensors belonging to a particular cluster send information of their Residual Energy (RE) and Mobility as shown in Figure 1. 

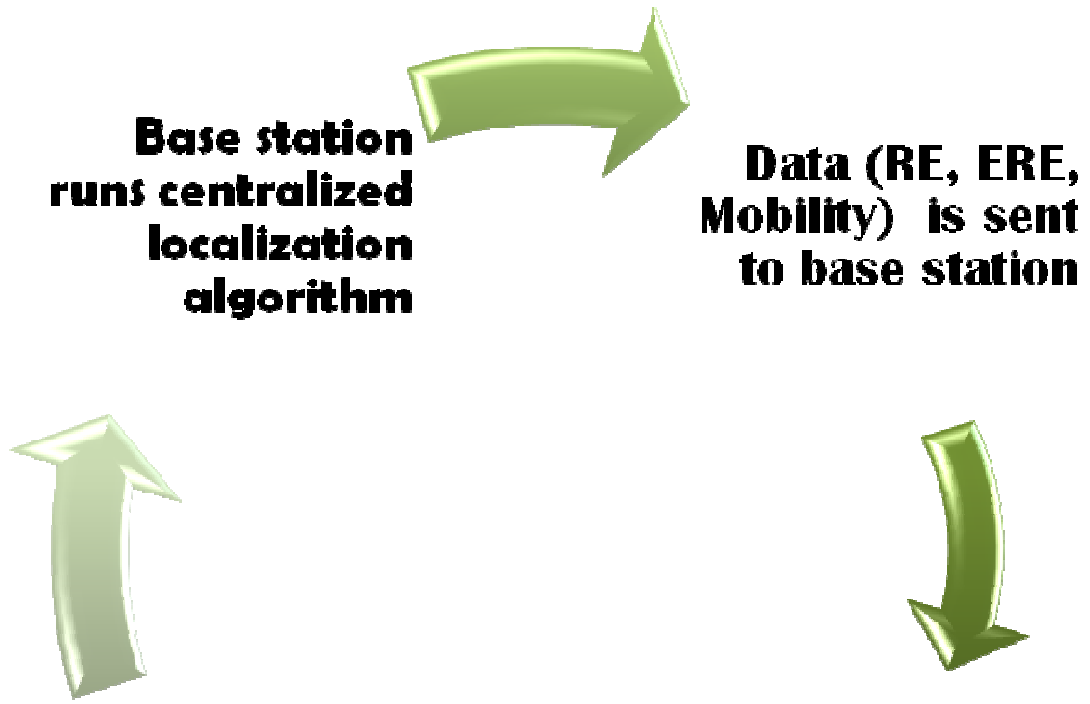

\title{
Node with highest chance becomes Cluster-head
}

\author{
Base stertion \\ runs CA-MWSN \\ algorithm
}

Fig 1. Round phases of CA-MWSN

On the basis of residual energy, Expected Residual energy (ERE) after next round is calculated. If ERE falls below Threshold energy, node is discarded from being competitor for cluster-head. ERE is a parameter for judging if a node can sustain for a round as a c luster-head. The Threshold Energy is the minimum energy required by a node to run a cluster-head round. Although, Threshold Energy is variable because it depends on number of nodes forming a cluster and distance of node from base station, however in the interest of maintaining simplicity of algorithm it can be considered as fixed amount of energy. Base station calculates Expected Residual Energy by following formula for round ' $r$ ':

Expected Residual Energy $(r)=$ Residual Energy $(r)-$ Energy Consumption $(r)$

Where Energy Consumption is calculated on the basis that if node acts as cluster-head. Energy Consumption in round ' $r$ ' depends on the location of sensor and the number of nodes in cluster. The distance between sensor and base station is followed by free space model [15]. The sensor node far from base station consumes more energy a s well as node with more number of nodes in cluster consumes more energy. I $n$ this way, the node with more ERE is stronger candidate for the selection of cluster head. 
International Journal on Applications of Graph Theory in Wireless Ad hoc Networks and Sensor Networks(GRAPH-HOC) Vol.6, No.4, December 2014

\section{THE PROPOSED ALGORITHM CA-MWSN}

CA-MWSN is an algorithm designed for mobile wireless sensor network which uses fuzzy logic to find th e cluster-head. There are three fuzzy descriptor Residual energy, Expected Residua 1 Energy and Mobility with which fuzzy inferen ce engine deals to decide 'Chance' of a node for being cluster-head. The node with highest 'Chance' is selected as c luster-head. Cluster-head works for a round and after completion of round becomes a general node and again participate in the election for cluster-head. The CA-MWSN algorithm is depicted below:

A-Total area

Ai- Sub-area

$\mathrm{N}$ - Total number of nodes

P- Total number of nodes in sub-area

RE- Residual Energy

ERE- Expected Residual Energy

EC- Energy Consumption

TH- Threshold Energy

M- Mobility

BS- Base station

$\mathrm{X}$ - X-coordinate of node

$Y$ - y-coordinate of node

FIE- Fuzzy Inference Engine

$\mathrm{CH}$ - Cluster head

THE CALCULATIONS ARE PERFORMED AT THE BASE STATION WHICH IS CONSIDERED AS SOURCE OF INFINITE ENERGY.

\section{ALGORITHM 1. CA-MWSN (A, N)}

1 for (i: $=1$ to $\mathrm{N})$

\{

2 Send i $(\mathrm{X}, \mathrm{Y})->\mathrm{BS}$

3 i++

$4 \quad$ BS divides A into $A_{j} \ldots \ldots \ldots . . . A_{k}$

5 for $A_{j}(j:=1$ to $k)$

\{

6 for $\mathrm{A}_{\mathrm{jk}}(\mathrm{k}:=1$ to $\mathrm{P})$

$7 \quad$ Send $A_{1 k}(R E, M)->B S$

$8 \quad \operatorname{ERE}\left(\mathrm{A}_{1 \mathrm{k}}\right):=\operatorname{RE}\left(\mathrm{A}_{1 \mathrm{k}}\right)-\mathrm{EC}$

9 If $\left(\operatorname{ERE}\left(\mathrm{A}_{1 \mathrm{k}}\right)\right)<\mathrm{TH}$

$10 \mathrm{k}++$

11 else

$12 \operatorname{FIE}\left(\operatorname{ERE}\left(\mathrm{A}_{1 \mathrm{k}}\right), \operatorname{RE}\left(\mathrm{A}_{1 \mathrm{k}}\right), \mathrm{M}\left(\mathrm{A}_{1 \mathrm{k}}\right)\right):=\mathrm{x}[\mathrm{k}]$

$13 \mathrm{k}++$

$14 \quad \operatorname{Max} x[\mathrm{k}]$

$15 \quad \mathrm{k} \rightarrow \mathrm{CH}$ of $\mathrm{A}_{1}$

$16 \mathrm{j}++$

\} 
International Journal on Applications of Graph Theory in Wireless Ad hoc Networks and Sensor Networks(GRAPH-HOC) Vol.6, No.4, December 2014

\}

\section{CONCLUSIONS AND FUTURE WORK}

This algorithm is able to satisfy the rational of proper coverage which is quite difficult in case of mobile sensor nodes. Location sensor nodes are considered before selection of cluster-heads whereby energy balance of all nodes can be maintained. However, execution of this algorithm can result in selection of two cluster-heads which may reside quite nearer to each -other, though residing in different sub-areas. Ergo, removal of this drawback is subsequent prospective of this work. In future, this very algorithm can be augmented with the selection of optimal fuzzy set and back-up cluster head. Choosing speed of nodes with random probability can be replaced with different walk models. Multi-hop architecture can also be supported with CA-MWSN in coming time.

\section{ACKNOWLEDGEMENTS}

The authors would like to thanks Ravi Kumar Gupta, Sachin, Shashi Ranjan and Shashi Shekhar for their valuable assistance.

\section{REFERENCES}

[1] Akcan, Hüseyin, et al. 2006. GPS-Free node localization in mobile wireless sensor networks. In Proceedings of the 5th ACM international workshop on Data engineering for wireless and mobile access. ACM Press, New York, NY, 35-42.

[2] Ren Y. Oleshchuk V. Li F. and Ge X. 2011. Security in mobile wireless sensor networks-a survey. Journal of Communications 6, 2 (Apr. 2011), 128-142.

[3] Giuseppe Anastasi, Marco Conti, Mario Di Francesco, Andrea Passarella, "Energy Conservation in Wireless Sensor Networks: a Survey”, Elsevier Ad Hoc Networks Journal, Vol. 7, No. 3, pp. 537-568, May 2009.

[4] Spencer BF, Ruiz-Sandoval M, Kurata N. "Smart Sensing Technology for Structural Health monitoring." Proceedings of the 13th World Conference on Earthquake Engineering, Vancouver,B.C., Canada, Paper No. 1791. 2004.

[5] Gizem, Aksahya \& Ayese, Ozcan (2009) Coomunications \& Networks, Network Books, ABC Publishers.

[6] Xing, G., Wang, X., Zhang, Y., Lu, C., Pless, R., and Gill,C. Integrated coverage and connectivity configuration and energy conservation in sensor networks. ACM Trans. on Sensor Networks, 1, 1 (Aug. 2005), 36-72.

[7] Heinzelman, W.R.; Chandrakasan, A.; Balakrishnan, H., "Energy-efficient communication protocol for wireless microsensor networks," System Sciences, 2000. Proceedings of the 33rd Annual Hawaii International Conference on , vol., no., pp.10 pp. vol.2, 4-7 Jan. 2000.

[8] Patwari, N.; Ash, J.N.; Kyperountas, S.; Hero, AO.; Moses, R.L.; Correal, N.S., "Locating the nodes: cooperative localization in wireless sensor networks," Signal Processing Magazine, IEEE , vol.22, no.4, pp.54,69, July 2005.

[9] Do-Seong Kim, Yeong-Jee Chung, "Self-Organization Routing Protocol Supporting Mobile Nodes for Wireless Sensor Network", in Proc. Computational Sciences IMSCCS, 2006, pp.622-626.

[10] G. Santhosh Kumar, Vinu Paul M V, K. Poulose Jacob, Mobility Metric based LEACH-Mobile Protocol, 16th International Conference on Advanced Computing and Communications, 2008 IEEE.

[11] C.-M. Liu, C.-H. Lee, L.-C. Wang, Power-efficient communication algorithms for wireless mobile sensor networks, in: Proceedings of the 1st ACM International Workshop on Performance Evaluation of Wireless Ad hoc, Sensor, and Ubiquitous Networks, 2004, pp. 121-122. 
International Journal on Applications of Graph Theory in Wireless Ad hoc Networks and Sensor Networks(GRAPH-HOC) Vol.6, No.4, December 2014

[12] Chuan-Ming Liu, Chuan-Hsiu Lee, Li-Chun Wang, Distributed clustering algorithms for datagathering in wireless mobile sensor networks, J. Parallel Distrib. Comput. 67 (2007) 1187 - 1200, Elsevier.

[13] Savazzi, P.; Favalli, L., "Dynamic Cell Sectorization Using Clustering Algorithms," Vehicular Technology Conference, 2007. VTC2007-Spring. IEEE 65th, vol., no., pp.604, 608, 22-25 April 2007.

[14] Nekooei, S.M.; Manzuri-Shalmani, M. -T, "Location Finding in Wireless Sensor Network Based on Soft Computing Methods," Control, Automation and Systems Engineering (CASE), 2011 International Conference, vol., no., pp.1, 5, 30-31July 2011.

[15] T. S. Rappaport, Wireless Communications: Principles and Practice, 2nd ed. Singapore: Pearson Education, Inc., 2002.

\section{Authors}

Ms. Priti Kumari research focuses on wireless sensor network particularly in area of energy conservation. Currently, she is working in designing localization algorithm for mobile wireless sensor network. Security aspects are also being considered. 